\title{
NEUMONIA POR PNEUMOCYSTIS: REPORTE DE UN CASO DE EVOLUCION FULMINANTE Y ACTUALIZACION DE SU ETIOLOGIA
}

\author{
Drs. Jaime Drewes $A^{(1)}$, Andrés Labra $W^{(2)}$, Javier Tenorio $P^{(2)}$.
}

1. Servicio de Radiología, CDT Hospital Barros Luco Trudeau.

2. Becados Radiología Universidad de Santiago de Chile.

\begin{abstract}
The case of a masculine patient, HIV positive with Pneumocystis Pneumonia (PCP), and a worse evolution is presented. The findings in thorax radiography as in CT are discussed. Literature is reviewed with emphasis in the last biologic findings in relation with Pneumocystis.

Key words: Pneumocystis jiroveci, Pneumocystis pneumonia, Pneumonia.
\end{abstract}

Resumen. Se presenta el caso de un paciente masculino, VIH positivo con neumonía por Pneumocystis y una evolución tórpida. Se discuten los hallazgos tanto en la radiografía simple como en la TC de tórax. Se revisa la literatura, poniendo énfasis en las últimas descripciones biológicas con relación al Pneumocystis.

Palabras claves: Neumonía, Neumonía por Pneumocystis, Pneumocystis jiroveci.

\section{Introducción}

La neumonía por Pneumocystis carinii, persiste como una de las infecciones oportunistas de mayor prevalencia en pacientes infectados con el virus de inmunodeficiencia humana (VIH). Descrito hace aproximadamente cien años como un protozoo fue reclasificado en 1988 como un hongo. El análisis de DNA demostró una gran diversidad de tipo de Pneumocystis en una estrecha relación huéspedespecie. Es así como se demostró que el Pneumocystis que ocasiona neumonía en humanos correspondía al "Pneumocystis jiroveci", descrito en 1999.

Revisaremos un caso clínico de curso poco habitual, como asimismo el agente etiológico, sus características biológicas, y las manifestaciones

Drewes J, y cols. Neumonia por pneumocystis: Reporte de un caso de evolución fulminante y actualización de su etiología. Rev Chil Radiol 2004; 10: 172-175.

Correspondencia: Dr. Jaime Drewes A.

jdrewesa@hotmail.com imagenológicas más frecuentes, tanto en radiología convencional como en tomografía computada (TC).

\section{Caso Clínico}

Hombre de 30 años con historia de disnea progresiva, sensación febril, tos seca, sudoración nocturna, baja de peso de aproximadamente $10 \mathrm{Kgs}$ y disfagia desde aproximadamente un mes. Con antecedentes de haber padecido varicela y gonorrea, ser homosexual e hijo de padre diabético. Al examen físico se encuentra un paciente de constitución ectomórfica, algo desnutrido (IMC: 18), sudoroso, pálido, con adenopatías cervicales e inguinales, F.R. de 52 por min, saturación de $92 \%$ con una $\mathrm{FiO} 2$ de $24 \%$, temperatura de $37^{\circ} \mathrm{C}$, retracción supraclavicular, respiración tóracoabdominal y estertores bibasales. De los exámenes de laboratorio destaca un hematocrito normal, gases sanguíneos con una hipoxemia leve, LDH: 833, baciloscopías ${ }^{(2)}$ : negativas, $\mathrm{VIH}$ : positivo y recuento de linfocitos CD4 $<200$ células $/ \mathrm{mm}^{3}$.

Su evolución clínica es tórpida presentando al cabo de 7 días, una falla respiratoria aguda requiriendo ventilación mecánica, falleciendo posteriormente.

El estudio radiológico de tórax demostró un progresivo compromiso pulmonar (Figuras 1,2) que motivó al sexto día de ingreso una TC (Figura 3). La evolución destacó por un importante compromiso destructivo pulmonar, pasando rápidamente desde un compromiso intersticial inicial a la destrucción del parénquima con formación de quistes y desarrollo de neumotórax.

\section{Discusión}

La enfermedad conocida hasta ahora como "Neumonía por Pneumocystis carinii" es una de las entidades que causan enfermedad y muerte en personas con inmunodeficiencia. Por largo tiempo ha sido la infección oportunista más común en pacientes con SIDA. 


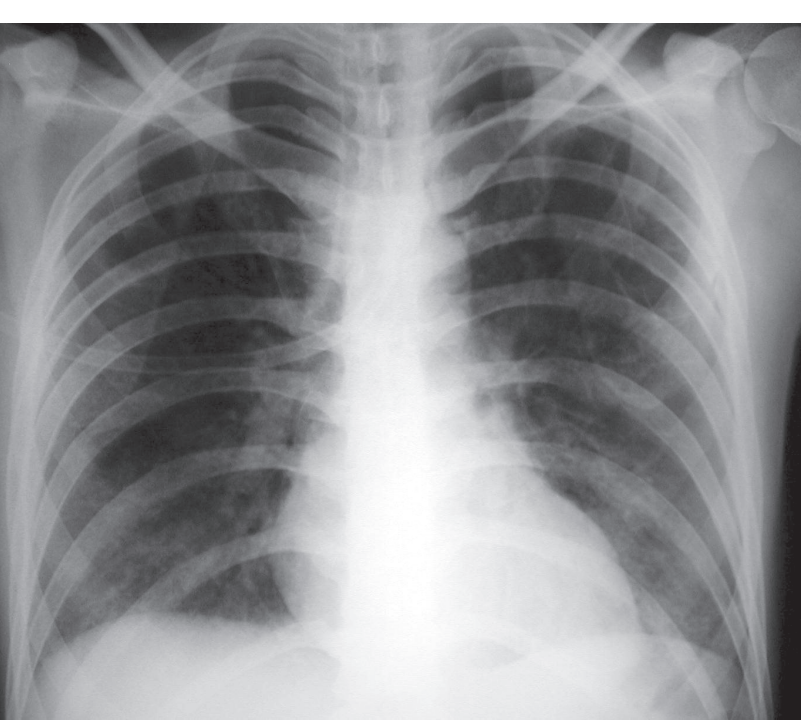

Figura 1. Radiografía de tórax obtenida al ingreso. Se demuestra un compromiso intersticial bilateral, de predominio perihiliar y basal, mayor a izquierda.
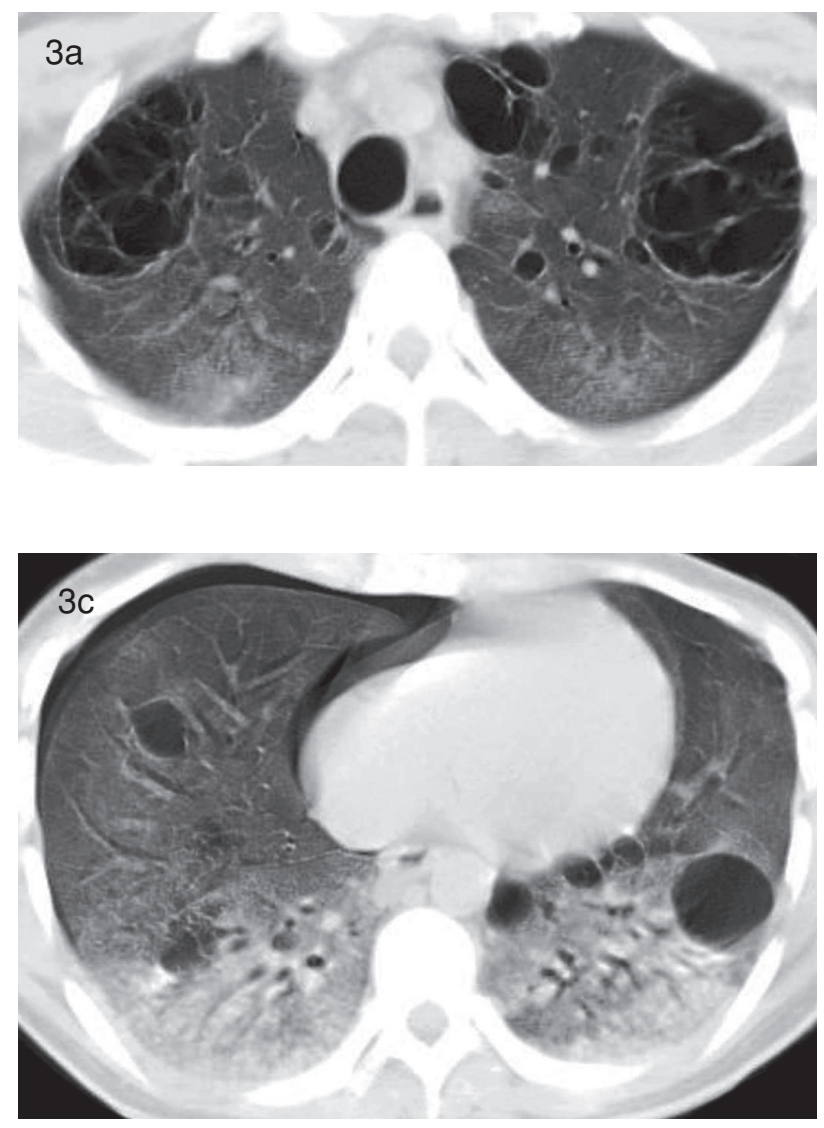

La introducción de la terapia antiretroviral altamente activa (HAART-highly active antiretroviral therapy), ha llevado a una sustancial reducción en la mortalidad y en la incidencia de infecciones oportunistas, incluyendo la neumonía por Pneumocystis $^{(1)}$.

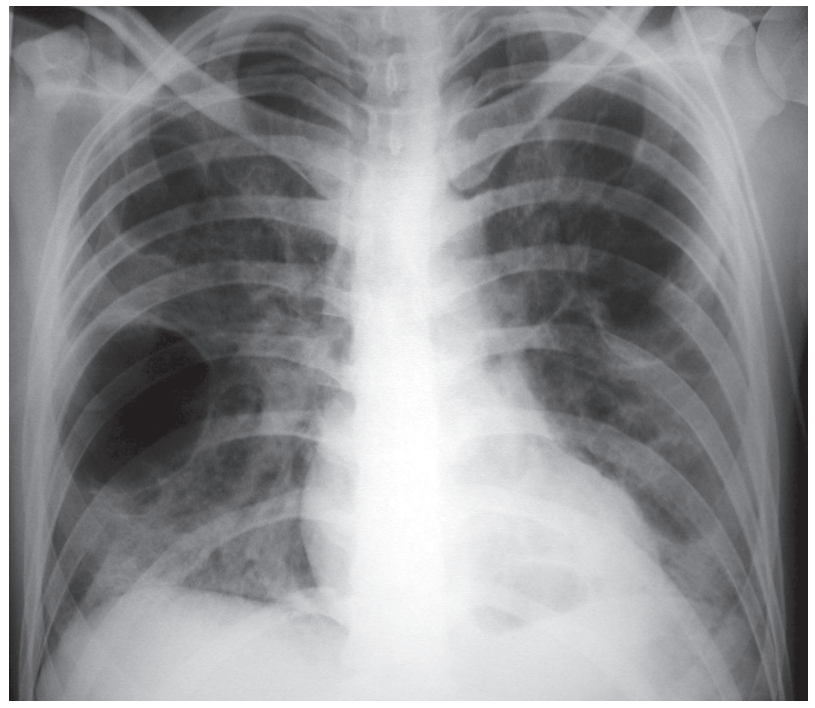

Figura 2. Radiografía de tórax control a los 5 días del ingreso. Se observa un aumento del compromiso intersticial, con opacidades tipo "vidrio esmerilado", asociado a imágenes quísticas en ambos hemitórax, predominantes en los lóbulos superiores.

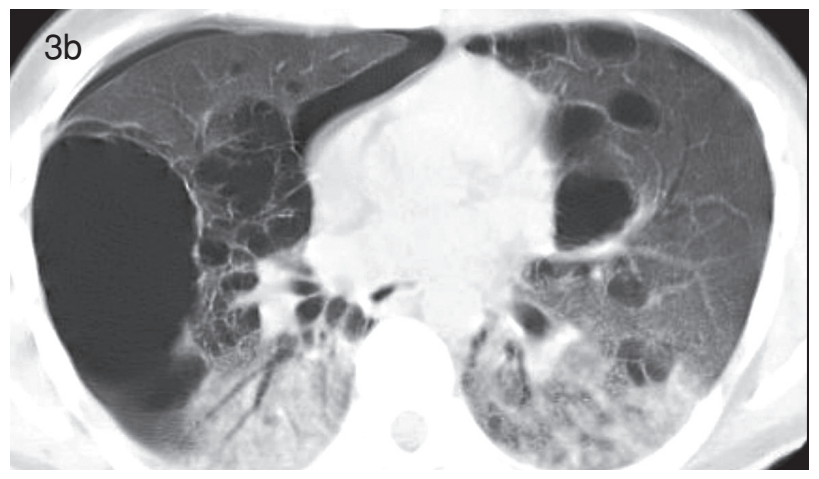

Figuras 3 a-c. TC de tórax realizada a los 6 días del ingreso. Se demuestra un patrón de vidrio esmerilado en prácticamente todo el parénquima pulmonar, áreas de condensación con broncograma aéreo principalmente en ambos lóbulos inferiores. También existen múltiples quistes aéreos de paredes finas de predominio subpleural, que tienden a coalescer. Neumotórax derecho.

A pesar de estos avances, el Pneumocystis permanece como el patógeno oportunista más importante en personas infectadas por $\mathrm{VIH}$ que no reciben o no responden al HAART, y en aquellos que ignoran su condición de infectados. Se estima que un $60 \%$ de los pacientes $\mathrm{VIH}$ positivos siguen infectándose por este agente en el transcurso de su enfermedad. Además, ella afecta a otros pacientes inmunocomprometidos, como lo son los trasplantados o quienes padecen de enfermedades malignas y están recibiendo quimioterapia. Recientemente se ha reportado infección por Pneumocystis en pacientes con inmunocompromiso 
moderado, incluyendo aquellos con enfermedad pulmonar crónica ${ }^{(1,2,4)}$.

Desde el punto de vista biológico la total identificación y clasificación del Pneumocystis tomó varias décadas. Se reportó por primera vez en 1909 por Carlos Chagas, como una forma de Tripanosoma cruzi. Luego fue descrito por Antonio Carinii en pulmones infectados por ratas. Ambos investigadores pensaron que habían identificado una nueva forma de tripanosoma, pero varios años más tarde se concluyó que se trataba de una nueva especie, por lo que se le llamó Pneumocystis carinii. Desde su descubrimiento hasta la década de los ochenta se clasificó como un protozoo, aunque algunos investigadores apuntaban a sus similitudes morfológicas con los hongos.

En 1988 un análisis de la subunidad rRNA en neumonía por Pneumocystis, estableció un enlace filogenético con hongos, a pesar de que no es posible realizar cultivos in vitro. Luego de su correcta clasificación el Pneumocystis se identificó en diferentes mamíferos, con características genéticas específicas para cada huésped. Años mas tarde, los estudios de análisis de DNA determinó que la especie que afecta a los humanos es diferente al de otros animales, lo que se comprueba en la secuencia $18 \mathrm{~S}$ rRNA del Pneumocystis jiroveci, derivado de los humanos y del Pneumocystis carinii derivado de las ratas, los que difieren aproximadamente en un $5 \%$ del total de su estructura proteica. Esta divergencia genética es típica del género que presenta una especificidad huésped-especie, siendo para el pneumocystis jiroveci imposible infectar ratas y para el carinii a los humanos. En 1999 se aceptó cambiar el nombre de la especie que infecta a humanos denominándola Pneumocystis jiroveci en honor al parasitólogo Checo, Otto Jirovec ${ }^{(2,3,5,6)}$.

Dada la evidencia de que la especie que afecta a humanos es diferente, se presentó el problema de su correcta designación en la literatura médica. Por consenso se estableció que el acrónimo usado en lengua inglesa "PCP" para describir el síndrome clínico de neumonía tanto en adultos como en mamíferos se mantendría, pero representando ahora PneumoCystis Pneumonia ("PCP") $)^{(2,3,5)}$.

El cuadro clínico más frecuente se da en pacientes con SIDA con recuento de linfocitos Tcolaboradores (CD4) menor de 200 células $/ \mathrm{mm}^{3}$. Los síntomas comunes incluyen disnea progresiva, tos no productiva, fiebre baja. La disnea aguda con dolor torácico puede indicar un neumotórax. El examen físico revela taquipnea, taquicardia y auscultación normal. Destaca dentro de los hallazgos en exámenes de laboratorio una LDH elevada, que es muy sensible en presencia de Pneumocystis, pero poco específica, por lo que tiene un valor limitado para el diagnóstico. Pacientes con SIDA y neumonía por pneumocystis jiroveci tienen un significativo aumento de microorganismos en los pulmones, con pocos neutrófilos, a diferencia de pacientes con esta enfermedad y no afectados por SIDA. Esto determina muy buenos resultados en el diagnóstico de esputo inducido y en broncoscopía con lavado broncoalveolar, donde se utiliza técnicas como PCR (polymerase chain reaction), detección de anticuerpos monoclonales y estudio directo con inmunofluorescencia. La biopsia transbronquial o quirúrgica son raramente requeridas ${ }^{(1,7)}$.

La mortalidad en pacientes con SIDA complicados con pneumonía por Pneumocytis jiroveci (PCP) oscila entre un $10-20 \%$, durante el periodo inicial de la infección, pero se ve incrementada si los pacientes llegan a las unidades de cuidados intensivos, con necesidad de ventilación mecánica como sucedió en nuestro caso $^{(4)}$. Se ha descrito que las formas lentamente progresivas de la infección, atípicas y con complicaciones como neumotórax, están asociadas a un significativo incremento en los decesos. Se calcula que la mortalidad se incrementa a un 75$100 \%$ en pacientes que no responden a la terapia durante los primeros 5-10 días ${ }^{(7)}$.

\section{Estudio radiológico}

El hallazgo más frecuentes en la radiografía de tórax es un compromiso intersticial de predominio perihiliar bilateral, que se va haciendo difuso y más homogéneo (Figura 4). También puede existir un compromiso de tipo alveolar o mixto, que junto al intersticial se presenta en un $85 \%$ de los casos, los hallazgos más característicos consisten en la presencia de opacidades reticulares o nodulillares tenues o áreas de consolidación irregular mal definido. En un 15\% de los pacientes la radiografía de tórax es normal.

En algunos casos pueden existir alteraciones quísticas con formación de neumatoceles, predominantes en los lóbulos superiores y con neumotórax asociado en pacientes con SIDA.

Los hallazgos más frecuentes en tomografía computada de alta resolución (TCAR) son la presencia de opacidades en vidrio esmerilado bilaterales, de distribución en parches o difusa, de predominio central y perihiliar o en los lóbulos superiores, cavidades septadas irregulares de paredes gruesas o quistes de paredes finas, neumotórax en relación a los quistes, como también superposición de todas las alteraciones descritas ${ }^{(6,7)}$ (Figuras 3). Estos hallazgos pueden reflejar el estadio de la enfermedad, distinguiéndose en la fase inicial focos dispersos de opacidades en vidrio esmerilado o consolidaciones, así como engrosamiento de los septos alveolares, lo que corresponde a la fase aguda de la enfermedad. En los pacientes en tratamiento o tratados se pueden distinguir opacidades reticulares, 

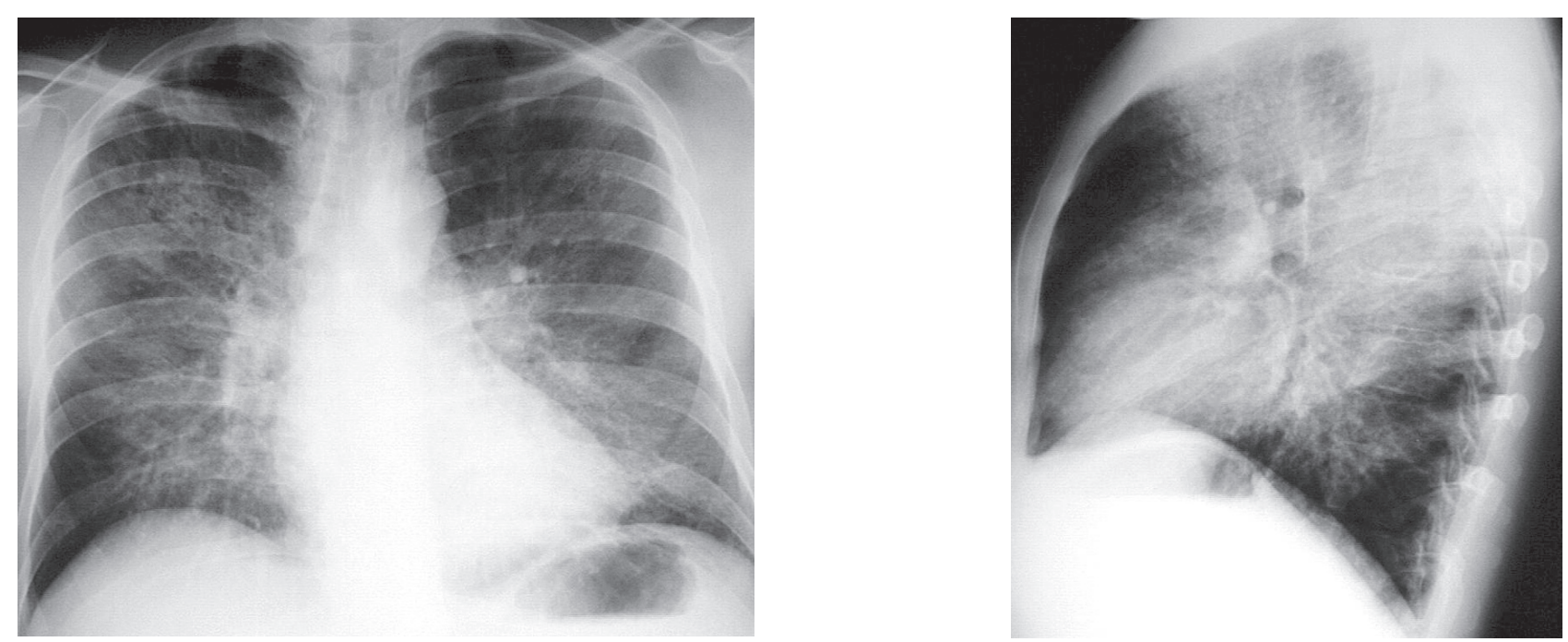

Figura 4 a,b. Radiografía de tórax PA (a) y L (b) en paciente VIH positivo con neumonía por Pneumocytis carinii confirmada. Se demuestra un compromiso intersticial bilateral, perihiliar tipo "vidrio esmerilado".

que traducen engrosamiento de los septos intralobulillares asociado a opacidades en vidrio esmerilado, lo que se conoce como fase subaguda o en resolución. La TCAR también es capaz de identificar los cambios residuales después del tratamiento, que pueden consistir en pequeñas áreas de consolidación, opacidades centrolobulillares, así como pequeñas bronquiectasias periféricas, probablemente secundario a bronquiolitis por infección por pneumocystis ${ }^{(6)}$.

El estudio radiológico en nuestro paciente quien presentó una evolución tórpida, demostró a su ingreso un patrón en vidrio esmerilado; que posteriormente se fue comprometiendo con cambios quísticos, de diferentes tamaños y formas, con paredes gruesas y finas, confluentes y subpleurales. Ello se correlaciona con la literatura en que existe una incidencia de aproximadamente $35 \%$ de este tipo de compromiso. Se ha descrito por algunos autores una enfermedad bulosa prematura en pacientes con SIDA, de distribución preferentemente en los lóbulos superiores y de localización subpleural, algunos de ellos con tendencia a coalescer formando quistes de formas irregulares y de paredes gruesas incluso con septos. Una vez en contacto con la pleura se pueden romper, lo que explica la alta incidencia de neumotórax en éstos casos. El neumotórax espontáneos asociado a la enfermedad quística pueden ser la primera manifestación radiográfica de un paciente con neumonía por pneumocystis. Este hallazgo en un paciente con SIDA es prácticamente diagnóstico de la enfermedad y sellan un pronóstico muy desfavorable ${ }^{(6)}$.

\section{Conclusión}

La neumonía por Pneumocystis sigue siendo la enfermedad oportunista más frecuente en pacientes inmunocomprometidos, en especial los que presentan SIDA. Actualmente la terminología que se debe emplear es neumonía por "Pneumocystis jiroveci”, ya que la neumonía por Pneumocystis carinii se presenta solo en ratas. La utilización del acrónimo "PCP" en ingles se sigue empleando ya que representa "PneumoCystis Pneumonia". Este caso grafica las consideraciones clínico-radiológicas en pacientes que no responden a la terapia antes de los 10 días del ingreso o que hacen neumotórax, ellos tienen un pronóstico francamente desfavorable, evolucionando con falla respiratoria e importante mortalidad.

\section{Bibliografía}

1. Thomas CF Jr, Limper AH. Pneumocystis pneumonia. N Engl J Med 2004; 350: 2487-98.

2. Stringer JR, Beard CB, Miller RF, Wakefield AE. A new name (Pneumocystis jiroveci) for Pneumocystis from humans. Emerg Infect Dis 2002; 8: 891-6.

3. Miller R. Pneumocystis pneumonia in humans is caused by $P$ jiroveci not $P$ carinii. Thorax 2004; 59: 83-84.

4. Nawaz A, Jones C, MacDonald S, Panigrahi H. Pneumonia, Pneumocystis Carinii. eMedicine 2003.

5. Miller R, Huang L. Pneumocystis jirovecii infection. Thorax 2004; 59: 731-733.

6. Totet A, Respaldiza N, Pautard JC et al. Pneumocystis jiroveci genotypes and primary infection. Clin Infect Dis 2003; 36: 1340-2.

7. Webb R, Müller N, Naidich D. High-Resolution CT of the Lung 3rd ed, Marbán Libros, S.L. 2003; páginas 396-403. 